\title{
ENHANCED GRAVITROPISM 2 encodes a STERILE ALPHA MOTIVE containing protein that controls root growth angle in barley and wheat
}

Authors : Gwendolyn K. Kirschner ${ }^{1}$, Serena Rosignoli ${ }^{2}$, Isaia Vardanega ${ }^{1,2}$, Li Guo ${ }^{1}$, Jafargholi Imani $^{3}$, Janine Altmüller ${ }^{4}$, Sara G. Milner ${ }^{7}$, Raffaella Balzano ${ }^{2}$, Kerstin A. Nagel ${ }^{5}$, Daniel Pflugfelder ${ }^{5}$, Cristian Forestan ${ }^{2}$, Riccardo Bovina ${ }^{2}$, Robert Koller ${ }^{5}$, Tyll G. Stöcker ${ }^{6}$, Martin Mascher $^{7,9}$, James Simmonds ${ }^{8}$, Cristobal Uauy ${ }^{8}$, Heiko Schoof ${ }^{6}$, Roberto Tuberosa ${ }^{2}$, Silvio Salvi ${ }^{2^{*}}$, Frank Hochholdinger ${ }^{1^{*}}$

${ }^{1}$ Institute of Crop Sciences and Resource Conservation (INRES), Crop Functional Genomics, University of Bonn, Germany

${ }^{2}$ Department of Agricultural and Food Sciences (DISTAL), University of Bologna, Italy

${ }^{3}$ Institute of Phytopathology, Research Centre for BioSystems, Land Use and Nutrition, JustusLiebig-University Giessen, Germany

${ }^{4}$ Cologne Center for Genomics (CCG), University of Cologne, Germany

${ }^{5}$ Institute of Bio- and Geosciences, IBG-2: Plant Sciences, Forschungszentrum Juelich GmbH, 52425 Jülich, Germany

${ }^{6}$ Institute of Crop Sciences and Resource Conservation (INRES), Crop Bioinformatics, University of Bonn, Germany

${ }^{7}$ Leibniz Institute of Plant Genetics and Crop Plant Research (IPK) Gatersleben, 06466 Seeland, Germany

${ }^{8}$ John Innes Centre, Norwich Research Park, Norwich NR4 7UH, United Kingdom

${ }^{9}$ German Centre for Integrative Biodiversity Research (iDiv) Halle-Jena-Leipzig, Leipzig, Germany

\section{${ }^{*}$ corresponding authors, email:}

hochholdinger@uni-bonn.de

silvio.salvi@unibo.it 


\section{Classification}

Biological Sciences

Plant Biology

\section{Keywords}

Barley, CRISPR/Cas9, EGT2, gravitropism, laser capture microdissection, root angle

\section{Author Contributions}

G.K.K., K.N., S.S., and F.H. conceived the study. C.F., G.K.K., D.P., I.V., J.A., J.I., J.S., L.G., R.Ba., R.Bo., S.G.M., and S.R. conducted the experiments. C.U., T.G.S., H.S., G.K.K, K.A.N., M.M., R.K., R.T., and S.S. analyzed data. G.K.K., F.H., S.R., and S.S. wrote the manuscript with inputs from all co-authors.

\section{This PDF file includes:}

Main Text

Figures 1 to 4

Supplementary Figures 1 to 7

Supplementary Tables 1 to 5 


\begin{abstract}
The root growth angle defines how roots grow towards the gravity vector and is among the most important determinants of root system architecture. It controls water uptake capacity, nutrient use efficiency, stress resilience and as a consequence yield of crop plants. We demonstrated that the egt2 (enhanced gravitropism 2) mutant of barley exhibits steeper root growth of seminal and lateral roots and an auxin independent higher responsiveness to gravity compared to wild type plants. We cloned the EGT2 gene by a combination of bulked segregant analysis and whole genome sequencing. Subsequent validation experiments by an independent CRISPR/Cas9 mutant allele demonstrated that egt2 encodes a STERILE ALPHA MOTIF domain containing protein. In situ hybridization experiments illustrated that EGT2 is expressed from the root cap to the elongation zone. Subcellular localization experiments revealed that EGT2 localizes to the nucleus and cytoplasm. We demonstrated the evolutionary conserved role of EGT2 in root growth angle control between barley and wheat by knocking out the EGT2 orthologs in the A and B genomes of tetraploid durum wheat. By combining laser capture microdissection with RNA-seq, we observed that seven expansin genes were transcriptionally downregulated in the elongation zone. This is consistent with a role of EGT2 in this region of the root where the effect of gravity sensing is executed by differential cell elongation. Our findings suggest that EGT2 is an evolutionary conserved regulator of root growth angle in barley and wheat that could be a valuable target for root-based crop improvement strategies in cereals.
\end{abstract}

\title{
Significance Statement
}

To date the potential of utilizing root traits in plant breeding remains largely untapped. In this study we cloned and characterized the ENHANCED GRAVITROPISM2 (EGT2) gene of barley that encodes a STERILE ALPHA MOTIF domain containing protein. We demonstrated that EGT2 is a key gene of root growth angle regulation in response to gravity which is conserved in barley and wheat and could be a promising target for crop improvement in cereals. 


\section{Main Text}

\section{Introduction}

The increase of human population and climate change are major challenges to food security (1, 2). A number of studies proposed to modify root system architecture to improve water and nutrient use efficiency, crop yield and resilience to stress episodes $(3,4)$. Among the most important determinants of root system architecture is the root growth angle, i.e. the angle in which roots grow towards the ground.

Increased response to gravity, or hypergravitropism, and thereby a steeper root growth angle was shown to be associated to improved drought resistance in rice, probably by increased access to deep-soil water (5). At the same time, a deeper root system facilitates the uptake of $\mathrm{N}$ and other mobile nutrients which are more abundant in deeper soil layers (6). Root gravitropism is regulated by sensing the gravitropic stimulus and subsequent differential cell elongation to enable root growth towards the gravitropic vector. Removing the root cap mechanically or genetically substantially diminishes the gravitropic response (7-9) suggesting that gravity sensing occurs primarily in the root cap. However, there is evidence for a sensing site outside the root cap, located in the elongation zone $(10,11)$. There are different hypotheses on how the cells sense gravity, with the prevailing idea that the starch-containing plastids in the root cap act as statoliths and settle in response to gravity. In doing so, they trigger a signaling cascade, either by mechanosensitive channels or by direct protein interaction, on the organelle surface (12-14). This signaling pathway ultimately leads to a rearrangement of auxin export carriers and thereby to a reorganization of the auxin maximum in the root tip (15). At the same time, changes of $\mathrm{pH}$ in the root cap and an asymmetrical change of $\mathrm{pH}$ in the upper and lower side of the root meristem and elongation zone occur $(16,17)$. This finally leads to an increased elongation of the cells on the side averted to the gravity vector in the elongation zone of the roots, so that the roots grow downwards (18). To date, only single components of the signaling cascade regulating root gravitropism have been unraveled. Examples include the actin-binding protein RICE MORPHOLOGY DETERMINANT that localizes to the surface of statoliths in rice root cap cells and controls the root growth angle in response to external phosphate (19). Another protein involved in gravitropism is the membrane-localized ALTERED RESPONSE TO GRAVITY1 in Arabidopsis, which is expressed in the root cap and is involved in the gravity-induced lateral auxin gradient (20). Both proteins seem to function in signaling immediately after gravity sensing in the root cap. In contrast, rice DEEPER ROOTING1 (DRO1), acts as early auxin response gene later in the gravitropic signaling. The DRO1 gene encodes for a plasma membrane protein that is expressed in the root meristem and was identified because of its influence on the root growth angle (5). The role of DRO1 may not be conserved in primary roots of different plant species, since the Arabidopsis homolog does not affect the gravitropic response of the primary root but influences the growth angle of the lateral roots (21).

Barley (Hordeum vulgare) is the world's fourth most important cereal crop in terms of grain production, after wheat (Triticum aestivum), rice (Oryza sativa) and maize (Zea mays) (2017, http://faostat.fao.org). It is cultivated over a broad geographical area because it can adapt to a wide range of climatic conditions and is therefore an excellent model to study responses to climate change (22). In this study we used a forward-genetics approach to clone ENHANCED GRAVITROPISM2 (EGT2), a novel gene involved in barley root gravitropic response and whose effect is conserved in wheat. EGT2 encodes a STERILE ALPHA MOTIF domain containing protein and likely acts in a regulatory pathway that counteracts the auxin-mediated positive gravitropic signaling pathway. 


\section{Results}

The egt2-1 mutant shows a steeper root growth correlated with an enhanced gravitropic response The egt2-1 mutant was discovered in a sodium-azide mutagenized population of the barley cultivar Morex based on the hypergravitropic growth of its seminal root system in paper rolls and shown to be inherited as a monogenic recessive Mendelian locus (23-25). We investigated the phenotype in more detail in 2-D rhizoboxes, in which the plants grow vertically on flat filter paper. While in Morex wild type the seminal roots grow in a shallow angle towards the gravity vector and cover a larger area, the seminal roots in the egt2-1 mutant grow steeply down (Figure 1A, Supplementary Figure 1A). This phenotype was consistent in plants grown in soil-filled rhizotrons and pots, the latter visualized by magnetic resonance imaging (MRI) (Figure 1B, C, Supplementary Figure 1E, F, G). Furthermore, the lateral roots arising from the seminal roots also displayed a highly increased growth angle (Figure 1B, E, Supplementary Figure 1A, H). Apart from the increased root growth angle, we did not detect any other aberrant root phenotypes, neither a changed number of seminal roots nor a difference in root length (Supplementary Figure $1 \mathrm{~B}, \mathrm{C})$. To further investigate the reason for the steep root phenotype, we tested the responsiveness of the root system to gravity. After rotation by $90^{\circ}$, we monitored the angle of the root tips over time (Figure 1F, G). Roots of the egt2-1 mutant bent much faster and stronger than wild type roots, approaching $90^{\circ}$ after 3 days compared to just $30^{\circ}$ in wild type roots (Figure $1 \mathrm{G}$ ). Root growth rate, however, was not altered (Supplementary Figure 1D). We concluded therefore that the steep root angle of the egt2-1 mutant was likely caused by a higher responsiveness to gravity. Since gravity sensing and signal transduction was shown to take place in the root cap and meristem (16-18), we compared the root cap and meristem by microscopy and measuring the root meristem size, but we did not discover significant differences (Supplementary Figure 2).

\section{Auxin response is unaffected in egt2-1 mutants}

It was shown before that the phytohormone auxin is involved in gravitropic response signaling (15) and that auxin transport inhibitors or external supply of auxin influences the reaction of roots to rotation (26). To analyze if the egt2-1 mutant is sensitive to manipulation of the auxin state in the roots, we treated wild type and mutant with auxin or auxin transport inhibitors and recorded the reaction to $90^{\circ}$ rotation. Application of the endogenous auxin Indole-3-acetic acid (IAA) as well as the synthetic auxin analog 1-Naphthaleneacetic acid (NAA) led to a faster, albeit nonsignificant, reaction in both wild type and egt2-1 mutant immediately after the rotation (Supplementary Figure 3A, C). Treatment with 2,4-Dichlorophenoxyacetic acid (2,4D), an auxin analog which cannot be transported by auxin efflux transporters, caused a significantly faster response in both wild type and egt2-1 mutant (Supplementary Figure 3E). Treatment with the auxin transport inhibitor 1-N-Naphthylphthalamic acid (NPA) on the other hand, decreased the reaction to rotation significantly in wild type and mutant to a similar degree (Supplementary Figure $3 G)$. Recently, cytokinin was described as anti-gravitropic signal in lateral roots, therefore we included cytokinin in our study (27). However, we found no impact of cytokinin on the root angle after rotation (Supplementary Figure 3I). In summary, we demonstrated that egt2-1 reacts to auxin treatments to the same degree as the wild type and we conclude that the mutation in egt2-1 does not disrupt the major auxin signaling pathways. This notion is consistent with the results of a tissue-specific RNA-seq analysis of wild-type and egt2-1 seminal roots where we did not find any auxin related genes among the differentially expressed genes (see results below).

EGT2 encodes a Sterile alpha motif domain-containing protein

In order to map and clone the EGT2 gene, a SNP-based bulked-segregant analysis (BSA) was carried out using an $\mathrm{F}_{2}$-population derived from the cross between the hypergravitropic egt2-1 carrying line TM2835 (in Morex background) and cv. Barke, the latter showing a typical wild-type, 
shallow root architecture. egt2 was mapped to a $312 \mathrm{Mbp}$ interval on the short arm of chromosome 5H (Figure 2A), between markers SCRI_RS_222345 and SCRI_RS_13395 (Table S1). Subsequently, TM2835 was subjected to whole genome sequencing, which led to the identification of seven genes within the egt2 interval and which carried missense, splice site or stop-codon gain mutations when compared with wild type Morex sequence (Table S2). Among these was a gene encoding for a 252 amino acid sterile alpha motif (SAM) domain-containing protein (HORVU5Hr1G027890 (24) or HORVU.MOREX.r2.5HG0370880.1 (28)) with a mutation (G447A) leading to a premature stop codon at the beginning of the functional domain (W149*) (Figure 2B, Supplementary Figure $4 \mathrm{~A}$ and B) (28). Apart from the SAM domain, no other functional domains were predicted (29). The sequence of the SAM domain between EGT2, and previously described SAM domains of other plant species is highly conserved (Supplementary Figure 4B, Supplementary Figure 5) $(30,31)$.

To validate HORVU.MOREX.r2.5HG0370880.1 as EGT2, we used CRISPR/Cas9 to create an additional mutant allele (egt2-2) in the barley cv Golden Promise. We targeted two sites in the 5'UTR and exon 1, separated by $196 \mathrm{bp}$, and recovered a $221 \mathrm{bp}$ deletion leading to a premature stop codon and a truncated 75 amino acid protein (Figure 2B , Supplementary Figure 4A). We analyzed the root phenotype of the homozygous $\mathrm{T}_{1}$-line and determined a significantly higher root angle of both seminal and lateral roots in the mutant in comparison to the wild type (Figure 2C, D, E, Supplementary Figure 4D). Hence, we confirmed that the altered root angle phenotype of egt22 is caused by a truncation of HORVU.MOREX.r2.5HG0370880.1. Like in the egt2-1 mutant in Morex background, the root length of egt2-2 was similar to the wild type (Supplementary Figure 4C). The reaction of the egt2-2 roots after rotation was faster than in the wild type, however, not statistically significant (Supplementary Figure 4E). It is notable that Golden Promise and Morex differ in seminal root angle growth although they both carry a wild-type EGT2 allele (compare Figure $1 \mathrm{~A}$ and $\mathrm{E}$ and Figure $2 \mathrm{C}$ and $\mathrm{D}$ ). Additionally, the re-orientation of the roots after rotation occurs much faster in wild type Golden Promise than in Morex (compare Figure $1 \mathrm{H}$ and Supplementary Figure 4E). Thus, other genetic factors influence the root growth angle in addition to EGT2.

To further validate the function of the EGT2 gene, we identified mutant lines carrying premature stop codons from a sequenced mutant population of tetraploid wheat (32). We combined mutations in the two durum wheat EGT2 orthologs (homologs on A and B genomes) to generate complete egt2 knockout lines. These double mutants showed narrower seminal root growth angle in rhizoboxes compared with the sibling lines carrying wildtype alleles in both homologs (Figure $2 F, G)$. Similar to barley, the number and length of seminal roots was unaffected in 7- day old seedlings (Supplemental Figure 4G, H, I).

EGT2 is expressed in the whole root tip and localizes to cytoplasm and nucleus

To survey the spatial expression patterns of EGT2 in roots we performed RNA in situ hybridization experiments. EGT2 is expressed in the whole root tip, including the root cap, meristem and elongation zone (Figure $3 \mathrm{~A}$ ). We confirmed this expression pattern by RNAseq data, where we found EGT2 expressed in root cap, meristem and elongation zone and a transcriptional downregulation only in the elongation zone of the mutant (Figure 3B). qRT-PCR analysis of combined root cap, meristem and elongation zone and the differentiation zone confirmed the expression in all these tissues in wild type and mutant (Figure 3C). Transient expression in Nicotiana benthamiana leaves revealed that EGT2 localizes to cytoplasm and nucleus (Figure 3D).

In the egt2-1 mutant, cell wall related processes are affected in the elongation zone

To analyze the effect of the mutation in EGT2 on the root transcriptome, we isolated RNA from different root tissues. For this, we applied laser capture microdissection to specifically separate root cap, meristem and parts of the elongation zone from wild type and egt2-1 seminal roots. This allowed us to differentiate between gravity sensing (root cap), signal transduction (meristem) and 
signal execution (elongation zone) (Figure 4B). To this end, we selected the most vertically grown seminal roots in both genotypes that displayed a similar root growth angle (Figure 4A). By doing so we excluded secondary effects caused by different root growth angles. Moreover, we used roots of similar length to exclude differences in age since the barley seminal roots do not grow out simultaneously (33). We determined the transcriptomic relationships among the two genotypes and three tissues by a principle component analysis (PCA) (Figure 4B). In the PCA, the two principle components PC1 and PC2 explained $82 \%$ of the total variance (Figure 4B). The biological replicates per tissue including four wild-type and four mutant samples clustered closely together. This indicates small transcriptomic differences between the genotypes but large differences between the tissues. To identify differentially regulated genes, we computed pairwise contrasts between the genotypes of the respective tissues (FDR $<5 \%$ and $\log _{2} F C>|1|$; see methods) for genes that uniquely mapped to chromosomes 1 to 7 (34). This resulted in 67 differentially regulated genes (DEGs) among all tissues, some of which were shared between all or two tissues (Supplementary Figure 6, Table S4). Strikingly, we found seven genes encoding for expansins downregulated in the elongation zone (Supplementary Figure 6, Supplementary Figure 7). Gene ontology (GO) terms were only assigned to genes downregulated in the elongation zone, all of them related to the term cell wall (Figure 4E). At the same time, this validates our data set, since expansins are expressed in the elongation zone and differentiated root tissue (35). Furthermore, we found that several genes categorized as peroxidase superfamily protein members, upregulated in either the meristem or the elongation zone (HORVU2Hr1G026420, HORVU7HR1G020300, HORVU3Hr1G036820) (Supplementary Figure 6). Differential regulation of a peroxidase superfamily protein-encoding gene was already found in a study in Arabidopsis related to agravitropic mutants (36). Moreover, we found that a gene encoding for calmodulin, a primary plant calcium receptor, downregulated in meristem and elongation zone (HORVU1Hr1G068440) (Supplementary Figure 6). Finally, we found a gene annotated as excocyst complex component 7 upregulated in the meristematic zone. Components of the exocyst are involved in directing exocytotic vesicles to fusion sites on the plasma membrane and might be involved in the distribution of the auxin transporter PINFORMED4 in Arabidopsis $(37,38)$. 


\section{Discussion}

The optimization of root system architecture has been recognized as one of the most important objectives in current breeding programs aimed at increasing resilience and sustainability of crops and agricultural systems $(4,39)$. Specifically, variation of root growth angle can affect the way roots explore different soil layers, capture nutrients and water and thus can influence drought tolerance, as shown for DRO1 in rice (4). However, knowledge about genes, gene interactions and regulatory networks in root development is currently limited in all major crops, including cereals.

Here we cloned a novel key regulator of root gravitropism, ENHANCED GRAVITROPISM2 (EGT2), in barley and wheat. Mutations in EGT2 lead to enhanced gravitropic response and thereby to a steeper root growth angle of seminal and lateral roots. We did not find any other root or shoot morphological trait affected by this mutation, indicating that EGT2 does not act in an ubiquitous signaling pathway, but rather is specific for root gravitropism. In Arabidopsis, most gravitropic mutants were discovered because they show agravitropic root phenotypes $(20,40)$. This is probably because a hypergravitropic phenotype is difficult to detect given that the primary root of Arabidopsis grows highly gravitropic in wild type and that Arabidopsis lacks seminal roots (41). In grasses, however, some mutants with hypergravitropic roots were discovered, for instance vIn2 and $r m$. The villin protein VLN2 facilitates microfilament bundling, while the actinbinding protein RMD links actin filaments with gravity-sensing organelles $(19,26)$.

The only predicted domain in EGT2 is the SAM domain. In animals, SAM domain containing proteins function as transcription factors, receptors, kinases or ER proteins (30). In plants, the best known protein containing a SAM domain is the transcription factor LEAFY (LFY) which is involved in flower and meristem identity formation. Modelling of Arabidopsis SAM proteins based on structure predictions and LFY characterization suggests that the majority of these proteins are able to form head-to-tail homo- or hetero-oligomers/polymers (30). The close phylogenetic relationship of EGT2 with AtSAM5 (At3g07760) indicates a similar potential of oligomerization for EGT2.

EGT2 is also closely related to WEEP, a SAM domain containing protein that was discovered because of the prominent shoot phenotype in peach tree mutants (31). Peach trees with deletions in WEEP show a weeping shoot growth phenotype, thus the branches grow in a wider angle and after gravistimulation by rotation by $90^{\circ}$, the branches do not orient their growth upwards again (31). Therefore, EGT2 and WEEP are likely involved in a similar pathway that regulates gravitropism, however in opposite directions of the plant growth. Bud grafting experiments in peach implied that WEEP encodes an autonomous determinant of shoot orientation for each branch, and that no mobile signals from other parts of the plants (like phytohormones) are necessary (31). Furthermore, no difference of auxin or abscisic acid concentration was detected in growing shoots between peach wild type and WEEP mutants, nor were genes associated with auxin biosynthesis or perception differentially expressed (31).

Similarly, we did not find any expression changes of genes related to auxin biosynthesis or perception in our transcriptomic comparison between wild type and egt2-1. Treatments with auxins or an auxin transport inhibitor confirmed that the egt2-1 mutant is as sensitive to disturbance of the auxin balance as the wild type (Supplementary Figure 3), indicating that EGT2 works independently of auxin. Nevertheless, the auxin transport pathway could still be affected, as demonstrated for the rice mutant villin2 (v/n2) that exhibits a disturbed recycling of the auxin efflux carrier PINFORMED2 (PIN2) and thereby a hypergravitropic root response (26). However, auxin treatment of pin2 mutants induced a restoration of the phenotype and the mutants were insensitive to auxin transport inhibitors which differs from egt2-1. On the other hand, exocyst 
complex component 7 was transcriptionally upregulated in the egt2-1 mutant. In Arabidopsis, disturbing the expression of EXOCYST7OA3 by knock-out or overexpression leads to a higher agravitropic response upon auxin efflux inhibitors probably by regulating the PIN4 localization in columella cells and thereby auxin distribution in root tips (37). It is conceivable that disturbance of expression levels in egt2-1 mutants might lead to a change in PIN localization and thereby a changed signal transduction, however, this hypothesis remains to be tested. The ubiquitous expression of EGT2 in root cap, meristem and elongation zone suggests a participation rather in the signal transduction of gravitropism than in the sensing or differential cell elongation.

Localization of EGT2 in cytoplasm and nucleus is similar to the predicted localization of the homolog AtSAM (30). In a split-ubiquitin yeast two-hybrid screen, AtSAM5 interacted with the calcium dependent protein kinase AtCPK13 (At3G51850) (42), putatively connecting AtSAM5 to calcium signaling pathways. Inhibition of the primary plant calcium receptor calmoldulin was shown to inhibit the response to gravity in Arabidopsis (40). In the egt2-1 mutant, Calmodulin 5 is transcriptionally downregulated in meristem and elongation zone, putatively connecting EGT2 with calcium-dependent signal transduction. It is still generally unknown, however, which role calcium plays in gravitropic signaling.

If we hypothesize a role for EGT2 in signal transduction, we would expect downstream targets in the elongation zone, where the effect of gravity sensing is executed by differential cell elongation (18). Here, we found a striking number of expansin genes transcriptionally downregulated (Supplementary Figure 6). Expansins are known as acid-induced cell wall loosening enzymes. However, most studies are based on the activity of bacterial enzymes and the function of expansins in plant cell walls is still unknown (35). Similarly, cell wall-related genes are differentially regulated in weep mutant peach trees, and the differentially regulated auxin response genes in weep mutants have roles in mediating cell expansion, or modulation of $\mathrm{H}^{+}$ transport (31). Besides expansins, we found three genes encoding peroxidase superfamily proteins upregulated in the meristem and elongation zone (Supplementary Figure 6). Downregulation of a peroxidase superfamily protein-encoding gene was already demonstrated in a study in Arabidopsis comparing inflorescence stems of wild type to scarecrow and short root mutant transcriptomes, which show no gravitropic response to rotation in the shoot (36). Peroxidases catalyze the consumption or release of $\mathrm{H}_{2} \mathrm{O}_{2}$ and reactive oxygen species (ROS). One class of peroxidases functions extracellularly, either for cell wall loosening or cell wall crosslinking (43), and the transcriptional regulation in egt2 might be related to the regulation of the expansins. Moreover it was shown that ROS work downstream of auxin signaling in root gravitropism, maybe as second messenger (44). Besides the auxin-mediated pathway that directs downward root growth, in Arabidopsis lateral roots, which develop a distinct gravitropic setpoint angle, a pathway was discovered that counteracts the gravitropic bending independently of auxin (27). It is based on cytokinin signaling, indicating that the gravitropic setpoint angle is set by a balance of this pathway and the auxin-mediated positive response to gravity.

Based on the broad expression pattern of EGT2 throughout root cap, meristem and elongation zone, and the interaction of the Arabidopsis AtSAM5 homolog with CPK13, we can hypothesize that $E G T 2$ is involved in the signal transduction of gravitropic signaling. The missing interference in auxin-related processes on the transcriptomic level and the susceptibility to auxin treatments implies that EGT2 is not involved in any signal transduction related to changes in auxin levels and/or transport. It is possible that EGT2 acts in a pathway that counteracts the auxin-mediated positive gravitropic signaling pathway, since for the growth in an angle towards the gravity vector, a pathway counteracting the positive reaction to gravity is needed. By knocking it out, the downward growth of the roots would dominate and create the hypergravitropic phenotype.

In summary our results suggest that EGT2 is an evolutionary conserved check point of seminal and lateral root growth angle in barley and wheat. EGT2 could be a promising target for rootbased crop improvement in cereals. 


\section{Materials and Methods}

\section{Plant material and growth conditions}

The egt2-1 mutation carrying line, TM2835, was derived from sodium azide mutagenesis of the cv. Morex as previously described $(24,25)$. For growth in rhizoboxes and on agar plates, the seeds were washed in $1.2 \%$ sodium hypochlorite for $5 \mathrm{~min}$ and rinsed with distilled water. Then they were incubated in darkness at $30^{\circ} \mathrm{C}$ over night to induce germination and only germinating seeds were used for further experiments. Growth in rhizoboxes for plant phenotyping and rotation experiments were conducted as described before (46). For phytohormone treatments, plants were grown on half-strength Hoagland solution (47), $\mathrm{pH} 5.8$, supplemented with $0.8 \%$ phytagel on square Petri dishes, which were placed in a $45^{\circ}$ angle. The plants were grown in growth cabinets (Conviron, Winnipeg, Manitoba, Canada) at $18^{\circ} \mathrm{C}$ at night $(8 \mathrm{~h})$ and $22{ }^{\circ} \mathrm{C}$ at day $(16 \mathrm{~h})$. For growth in rhizotrons filled with peat substrate, wild type and egt2 mutants were grown in the GrowScreen-Rhizo automated platform for 24 days as previously described (48). For the MRI measurements, the seeds were placed in a Petri dish on wet filter paper. The Petri dish was sealed with parafilm and stored lightproof for $24 \mathrm{~h}$ in the growth chamber $\left(16^{\circ} \mathrm{C} / 20^{\circ} \mathrm{C}\right.$ night/day temperature, $14 \mathrm{~h}$ light per day) to induce germination and only germinated seeds were used for further experiments. Seeds were subsequently sown in field soil (Sp2.1, Landwirtschaftliche Untersuchungs- und Forschungsanstalt, Speyer, Germany). Soil moisture was kept at $8.9 \% \mathrm{~m} / \mathrm{m}$, corresponding to $40 \%$ of the maximal water holding capacity (49). Per genotype, 18 seeds were planted in one pot $(\varnothing=12.5 \mathrm{~cm}, 12 \mathrm{~cm}$ height) in a hexagonal grid with $2.5 \mathrm{~cm}$ seed spacing. Seedlings were imaged after 3 days in the growth chamber. For a longer experiment, single seeds were planted into larger pots $(\varnothing=9 \mathrm{~cm}, 30 \mathrm{~cm}$ height) and were grown for one week before imaging.

Durum wheat (Triticum turgidum) egt2 mutants were identified from a TILLING population developed in tetraploid cv Kronos (32). Two selected lines (Kronos2138 and Kronos3589) carrying premature termination codons in the two EGT2 homoeologous coding sequences (TraesCS5A01G102000 and TraesCS5B02G164200LC) were crossed and $F_{1}$ plants were selfpollinated. Progenies of selected wild-type and double mutant $F_{2}$ individuals derived from two independent initial crosses were grown in rhizoboxes for seminal root angle analysis. Seeds were washed in $70 \%$ ethanol for $1 \mathrm{~min}$, then in $1 \%$ sodium hypochlorite $+0.02 \%$ TritonX-100 for $5 \mathrm{~min}$ and rinsed with distilled water. Sterilized seeds were pre-germinated for $24 \mathrm{~h}$ at $28^{\circ} \mathrm{C}$ in wet filter paper. Only germinating seeds were transferred in rhizoboxes for 7 days at $25^{\circ} \mathrm{C}$.

\section{Phenotyping experiments and rotation tests}

For analysis of the root angles, plants were grown in rhizoboxes for 7 (seminal root angle) or 14 days (lateral root angle). The seminal root angle was measured as angle from the shoot to the root tip, in relation to the horizontal. For the angle of the lateral roots, the angle was measured from the outgrowth point of the main root to the lateral root tip in comparison to the horizontal. 20 randomly chosen lateral roots were measured per plant. For the rotation tests, the plants were grown in rhizoboxes for 5 days and then rotated once by $90^{\circ}$. For phytohormone treatments, the plants were grown for 5 days on agar without phytohormones and then transferred to agar plates supplemented with phytohormones as indicated in the results. After $1 \mathrm{~h}$ recovery, the agar plates were rotated once by $90^{\circ}$. Pictures were taken at the time points indicated in the graphs. For analysis, the root angle of every single root tip was measured in relation to the horizontal and the angle right after rotation was set to 0 . For all measurements, the average of all roots per plant was calculated, presented in the graphs and compared in the statistical tests. For analysis of growth in the rhizotrons, root images were collected every two days, enabling to distinguish between seminal and crown roots. Images at 24 days were utilized for seminal, nodal and lateral root angle analysis. Root angle values were collected with the software ImageJ (50). 


\section{$\underline{\text { RNA in situ hybridizations }}$}

Probes for EGT2 (HORVU5Hr1G027890) mRNA were prepared from the whole coding sequence (start to stop codon). Cloning and RNA probe synthesis was performed as described before (33). RNA in situ hybridizations on roots of 7-day-old plants was performed as described before (33).

\section{Protein localisation in Nicotiana benthamiana}

Constructs for transient expression in tobacco ( $N$. benthamiana) were built using the greengate system, with the EGT2 (HORVU5Hr1G027890) CDS in the pGGC module, the 35S promoter in the pGGA module (pGGA004), the UBQ10 terminator (pGGE009) and hygromycin resistance in the pGGF005 vector (51). TagRFP or mVENUS were used as fluorophors (52), either in the pGGB module as $\mathrm{N}$-tag or in the pGGD module as C-tag (Table S5). The Agrobacterium tumefaciens strain GV3101 was transformed with expression clones and cultured in lysogeny broth medium. Bacterial cultures were precipitated and dissolved in $10 \mathrm{mM} \mathrm{MgCl} 2,10 \mathrm{mM} \mathrm{MES}$, and $100 \mu \mathrm{M}$ AS and infiltrated into tobacco leaves. Transgene expression was analyzed 3 days after infiltration.

\section{Bulked segregant analysis (BSA) and whole genome sequencing (WGS)}

BSA (53) was carried out using plants from an $F_{2}$-population obtained starting from the cross TM2835 $\times \mathrm{cv}$. Barke and segregating for the EGT2 locus. $106 \mathrm{~F}_{2}$-seedlings were grown in flat rhizoboxes composed by two black plastic panels of $38.5 \times 42.5 \mathrm{~cm}$. Five pre-germinated seeds ( 1 day, $20^{\circ} \mathrm{C}$, on wet filter paper, in the dark) were positioned between moist filter paper sheets within each rhizobox. Each rhizobox was placed vertically in a larger plastic tank containing deionized water to a level of $3 \mathrm{~cm}$ from the bottom, in growth chamber) at $18{ }^{\circ} \mathrm{C}$ at night $(8 \mathrm{~h})$ and $22{ }^{\circ} \mathrm{C}$ at day $(16 \mathrm{~h})$ for 13 days. At the end of the growing period, root growth angle of seedlings was visually evaluated and a segregation rate of 88:18 (wild-type vs. hypergravitropic) recorded confirming that egt2 segregates as a monogenic recessive Mendelian locus ( $\left.\chi^{2} 3: 1=n s\right)$ as previously described (25). Immediately after this inspection, 15 plants showing wild-type root growth angle and 15 plants showing an hypergravitropic angle were chosen for DNA preparation on a single plant basis using $2 \mathrm{~cm}^{2}$ leaf portions as previously described (45). DNA samples for BSA were obtained by mixing equal DNA amounts of each of the 15 bulk components, to a final concentration of $50 \mathrm{ng} / \mathrm{ul}$. The two DNA bulks (in double) along with single plant DNA samples from 10 hypergravitropic plants were genotyped using the 9k Illumina Infinium iSelect barley SNP array (54). SNPs signal was analyzed using GenomeStudio (Illumina, San Diego, Inc.). For DNA bulks, SNPs signal was interpreted using the theta value approach as described in (55), modified in order to integrate for each SNP the signals obtained from two bulks (wild type or $+/+$ and hypergravitropic or -/-) in the "delta theta" value score as follows "delta theta" $=[($ theta bulk $+/+)$ (theta bulk -/-)] $]^{2}$.

Genomic DNA of TM2835 for whole genome shot gun sequencing was extracted from leaf samples using a commercial kit (Macheray-Nagel Nucleospin $®$ Plant II). The DNA was sequenced with Illumina HiSeq PE150, and 699,353,963 paired-end reads were produced corresponding to a coverage of approx. 40x. Reads were aligned to the first version of barley $\mathrm{cv}$. Morex reference genome (24) with BWA v.7.12 (56) and variants in the genomic space were called with SAMtools v. $1.3(57,58)$, filtering for a minimum reads depth of $5 \times$, PHRED quality > 40. In order to discard background mutations due to the differences between the official Morex reference and the Morex parental seeds which had previously been used in the mutagenesis, the SNP calling considered further eight TILLMore mutants WGS data that was available at that moment, filtering with a custom AWK script for a minimum ratio DV/DP of 0.8 for the egt2 mutant and a maximum ratio of 0.2 in every other mutant, where DP is the coverage depth at the SNP position and DV is number of non-reference bases at the same position. SNP effects were predicted with SNPEff v.3.0.7 (59). 
For coverage analysis, a minimum of $5 \times$ read depth was considered, resulting in a target region of 3.5 GB containing a total of 15,805 mutations, hence the estimated mutation load on the entire genome is 22,579 mutations, or approx. 1 mutation per $220 \mathrm{~kb}$ which is of the same order of magnitude of mutation density (1 per $374 \mathrm{~kb}$ ) formerly estimated based on TILLING results from the same TILLMore population (24). For the provean analysis, values <-2.5 were considered deleterious and values $>-2.5$ were tolerated.

\section{Modified pseudo-Schiff propidium iodide (mPS-PI) and Lugol staining}

The mPS-PI staining was performed as described in (33). For Lugol staining, roots were fixed in $4 \%$ para-formaldehyde in phosphate buffered-saline (PBS) over night, embedded in $13 \%$ agarose and sectioned at the vibratome with $80 \mu \mathrm{m}$ thickness. Then they were stained with Lugol solution for $5 \mathrm{~min}$ and rinsed with water.

\section{Microscopy}

Transient expression in tobacco leaves was examined with a 25x Zeiss water-immersion objective using a Zeiss LSM 780 confocal microscopy system. tagRFP was excited with a $543 \mathrm{~nm}$ using a Helium Neon laser with emission detection through the meta-channel at 579 to $633 \mathrm{~nm}$, the laser power is $30 \%$. mVenus was excited at $488 \mathrm{~nm}$ by the argon laser with a $2.6 \%$ laser power, and emission was detected at 517 to $553 \mathrm{~nm}$ via the meta- channel (ChS2). DAPI was excited with a $405 \mathrm{~nm}$ using a diode laser, the laser power is $16 \%$, and emission was detected at 440-480 nm. RNA in situ hybridization and Lugol-stained samples were examined using a Zeiss PALM MicroBeam microscope.

$\underline{\mathrm{MRI}}$

$\mathrm{MRI}$ images were acquired on a $4.7 \mathrm{~T}$ vertical magnet equipped with a Varian console (60). A multi slice spin echo sequence was used. Sequence parameters were adapted to the different pot sizes. For the $9 \mathrm{~cm}$ pots, we used a birdcage RF coil with $10 \mathrm{~cm}$ diameter and the following sequence parameters: $0.5 \mathrm{~mm}$ resolution, $1 \mathrm{~mm}$ slice thickness, $9.6 \mathrm{~cm}$ field of view, TE $=9 \mathrm{~ms}$, $\mathrm{TR}=2.85 \mathrm{~s}$, Bandwidth $=156 \mathrm{kHz}, 2$ averages. For the $12.5 \mathrm{~cm}$ pots, following parameters were changed: birdcage RF coil with $140 \mathrm{~mm}$ diameter, $14 \mathrm{~cm}$ field of view, and $0.55 \mathrm{~mm}$ resolution.

\section{$\underline{\text { CRISPR }}$}

For CRISPR target sequences, we choose twenty base pair sequences with the protospacer adjacent motif PAM sequence NGG in the first exon of EGT2 (HORVU5Hr1G027890) that we checked at http://crispr.dbcls.jp/ for off-targets in the barley genome (Barley (Hordeum vulgare) genome, 082214v1 (Mar, 2012)). We used sites with only one predicted target for a 20mer and only up to 3 predicted targets for the 12mer target sequence upstream of the PAM. The CRISPR guide sequences are marked in Supplementary Figure 4A. The sgRNA shuttle vectors pMGE625 and 627 were used to generate the binary vector pMGE599 as described in (61). Transformation was carried out with the spring barley cv. Golden Promise grown in a climate chamber at $18{ }^{\circ} \mathrm{C} /$ $14^{\circ} \mathrm{C}$ (light/dark) with $65 \%$ relative humidity, with a $16 \mathrm{~h}$ photoperiod and a photon flux density of $240 \mu \mathrm{mol} \mathrm{m}-{ }^{2}$ sec- $^{1}$. The binary vector pMGE599 was introduced into Agrobacterium tumefaciens AGL-1 strain (62) through electroporation (E. coli Pulser; Bio-Rad, http://www.bio-rad.com). The scutella tissue of barley caryopsis was used for Agrobacterium-mediated transformation as described previously (63). The insert integration in the barley genome was confirmed by detection of hygromycin gene sequences via PCR in generated T0 lines and were analyzed for mutations in EGT2 by PCR and Sanger sequencing and the seeds for T1 generation were used for experiments (Table S5).

qRT-PCR 
For the qRT-PCR, RNA from plants grown for 7 days after germination in rhizoboxes was extracted with the RNeasy Plant Mini Kit (Qiagen) and first strand cDNA was synthesised with the RevertAid First Strand cDNA synthesis Kit (ThermoFisher). Ca. 12 plants were pooled for one biological replicate per sample and samples were divided into root tip (containing meristem and elongation zone, $\sim 2 \mathrm{~mm}$ until the outgrowth of root hairs) and adjacent differentiation zone $(\sim 8 \mathrm{~mm})$. For each genotype, 4 biological replicates and 3 technical replicates were used. For the reaction, $2 \mu$ of PerfeCTa SYBR Green SuperMix (Quantabio), $1 \mu$ l primer mix of a concentration of $1 \mu \mathrm{M}$ and $1 \mu \mathrm{l}$ cDNA was mixed. The primer efficiency of each oligonucleotide was calculated using the following dilution series: 1, 1/2, 1/4, 1/8,1/16, 1/32, 1/64, and 1/128. The relative expression levels of the transcripts were calculated with reference to the housekeeping gene tubulin (HORVU1Hr1G081280) and according to the method described in (64). Significant differences in gene expression levels were determined by a two-sided Student's t-test.

\section{Laser capture dissection microscopy (LCM) and RNAseg}

Root tips of the most vertically grown seminal root of 3-day-old plants were used and assigned as one biological replicate. Per genotype, 4 biological replicates were analyzed. Plants were grown in rhizoboxes and fixed with Farmers fixative (EtOH:Acetic acid 3:1) on ice for 15 min under 500 mbar vacuum and subsequent swirling at $4{ }^{\circ} \mathrm{C}$ for $1 \mathrm{~h}$. The fixation solution was replaced and the procedure was repeated twice before replacing the solution with $34 \%$ sucrose and $0.01 \%$ safranin-O in PBS. The samples were vacuum-infiltrated again for $45 \mathrm{~min}$ and incubated on ice at $4^{\circ} \mathrm{C}$ for $21 \mathrm{~h}$. Then the samples were dried carefully with tissue paper and embedded in tissuefreezing medium as described before (65). The medium blocks containing the tissue were stored at $-80^{\circ} \mathrm{C}$ and were acclimatized to $-20^{\circ} \mathrm{C}$ in the cryomicrotome (Leica CM1850). Longitudinal sections of $20 \mu \mathrm{m}$ thickness were mounted on poly-L-lysine-coated glass slides (Zeiss) and the tissue-freezing medium was removed after 5:30 min by incubation in $50 \% \mathrm{EtOH}$ and $1 \mathrm{~min}$ incubation each in $70 \% \mathrm{EtOH}, 95 \% \mathrm{EtOH}, 100 \% \mathrm{EtOH}$ and $100 \%$ xylol (RNase-free). The tissues (root cap, meristem and $900 \mu \mathrm{m}$ of the elongation zone adjacent to the meristem) were cut with the following settings of the PALM Microbeam laser capture instrument (Zeiss, Germany): Energy: 79, speed: 100, Cutting Program: "Center RoboLPC", picked up manually with a sharp needle and transferred to the cap of RNAse-free adhesive caps (Zeiss). RNA was isolated with the Arcturus PicoPure RNA Isolation Kit (Thermo Fisher) according to the manufacturer's protocol for tissue, including the DNase treatment. RNA quality was determined with an Agilent 2100 Bioanalyzer using the Agilent RNA 6000 Pico kit and yielded RIN values between 7.1 and 8.9 and a concentration between 610 and $95.000 \mathrm{pg} / \mu \mathrm{l}$ (Table S3). Pre-amplification and library preparation was carried out as described in (66). Detection and sequenced on an lllumina NovaSeq sequencing instrument with a PE100 protocol. The RNA-Seq experiments yielded on average 41 million 100 bp paired-end reads per sample (Table S3). Trimmomatic version 0.39 (67) was used to remove low-quality reads and remaining adapter sequences from each read dataset. Specifically, a sliding window approach was used, in which a read was clipped if the average quality in a window of $4 \mathrm{bp}$ fell below a phred quality score of 15 . Only reads with a length of $\geq 30 \square \mathrm{bp}$ were retained for further analyses. Data are deposited at the sequence read archive (SRA), PRJNA589222. BBDuk of the BBTools suite (https://jgi.doe.gov/data-andtools/bbtools/) was employed to remove rRNA reads from the datasets using a kmer length of 27 as filtering threshold for decontamination. After removal of rRNA reads, on average 8 million paired reads remained. The splice-aware STAR aligner v.2.7.2b (68) was used to align the remaining reads against a genome index of the barley reference sequence and annotation of genotype Morex (IBSC v2.0) (24). Multi-mapping reads that mapped to more than one position were excluded from subsequent steps by considering only reads, which mapped in a single location (--outFilterMultimapNmax 1). On average 5 million reads per sample aligned to unique positions in the gene set of the IBSC v2.0 barley reference genome with 46,272 predicted coding and non-coding gene models (EnsembIPlants release 45, (24), Table S3). The aligned paired-end 
reads were ordered according to their position and transformed to .bam files by the software samtools (version 1.3.1, (57)). Alignment of sequences to the reference genome of Morex (release 45) (24) was performed using HTSeq (version 0.10.0, (69)) with the parameters '-r pos -i gene_id -s no --secondary-alignments ignore --supplementary-alignments ignore'. The principal component analysis (PCA) was performed on the expression data using the normalization procedure $r \log ()$ implemented in the $\mathrm{R}$ package DESeq2 and the plotPCA() function (version 1.22.2, (34)). Expression values were normalized with library size by calculating fragments per million (FPM) reads using the fpm() function of DESeq2, after removal of lowly expressed genes with less than 10 reads over all samples. Expression levels of genes were estimated by the variance-mean dependence in the count table based on a generalized linear model using the negative binomial distribution within the $R$ package DESeq2 (34) calculating $\log _{2}$ fold change $\left(\log _{2} \mathrm{FC}\right)$ values between wild type and mutant in the respective tissues with the design genotype + tissue + genotype:tissue. Significance values for $\log _{2} \mathrm{FC}$ values were calculated as Wald test $p$-value and were adjusted by the Benjamini-Hochberg procedure to obtain false discovery rates (FDR) (70). Genes with a FDR $<5 \%$ and $\log _{2} F C$ of $|1|$ were considered differentially expressed. From this gene set, we excluded gene pairs that were assigned to chr0 and chr1 which had the same annotation and the respective gene partner was the one with the closest related transcript after a BLAST search. Gene ontology term enrichment of the resulting gene set was performed using agriGo (71). The sequencing data have been deposited in the $\mathrm{NCBI}$ sequencing read archive (PRJNA589222).

\section{Phylogenetic analysis}

The EGT2 (HORVU.MOREX.r2.5HG0370880.1) protein sequence was BLASTed on Phytozome v12.1 to the Brachypodium dystachyon proteome v3.1, the Oryza sativa proteome v7_JGl, the Zea mays proteome Ensembl-18, the Arabidopsis thaliana proteome TAIR10, the Prunus persica proteome v2.1 and the Sorghum bicolor proteome v3.1.1 with the default settings. Hits with Evalues $<3.9 \mathrm{E}-77$ were considered. The identified orthologs were then confirmed using the EnsembIPlants Compara Ortholog tool. Retrieved protein sequences were aligned by ClustalW in the software MEGA X, with default values (72): Ancestral states were inferred using the Maximum Likelihood method (73) and JTT matrix-based model (74). The tree shows a set of possible amino acids (states) at each ancestral node based on their inferred likelihood at site 1 . The initial tree was inferred automatically by applying Neighbor-Join and BioNJ algorithms to a matrix of pairwise distances estimated using the JTT model, and then selecting the topology with superior log likelihood value. The rates among sites were treated as being uniform among sites (Uniform rates option). This analysis involved 16 amino acid sequences. 


\section{Acknowledgments}

This work was funded by the Deutsche Forschungsgemeinschaft (DFG) grant HO2249/21-1 to FH. KAN, DF and RK acknowledge support from the Helmholtz Association for the Forschungszentrum Jülich. The rhizotron study received funding from the European Union's Horizon 2020 research and innovation programme under grant agreement No 731013 (EPPN2020). Work described here is supported in part by the project 'Rooty- A root ideotype toolbox to support improved wheat yields' funded by the IWYP Consortium (project IWYP122) to $\mathrm{CU}, \mathrm{JS}, \mathrm{RT}$ and SS, via the Biotechnology and Biological Sciences Research Council in the United Kingdom (BB/S012826/1).

The authors thank Dr. Felix Frey (University of Bonn) for his advice on RNAseq data analysis and discussion, Dr. Johannes Stuttmann (University of Halle) for sharing the CRISPR/Cas cloning vectors and Shalima H. Orse for support throughout the project. We thank Anna Galinski, Jonas Lentz, Carmen Müller, Bernd Kastenholz, Ann-Katrin Kleinert, Roberta Rossi, and Kwabena Agyei (Forschungszentrum Jülich $\mathrm{GmbH}$ ) for their assistance during the rhizotron study. RK and DP gratefully acknowledge Dagmar van Dusschoten and Johannes Kochs for support and maintenance of the MRI System. 


\section{References}

1. T. Wheeler, J. von Braun, Climate Change Impacts on Global Food Security. Science (80.). 341, 508-513 (2013).

2. N. Ramankutty, et al., Trends in Global Agricultural Land Use: Implications for Environmental Health and Food Security. Annu. Rev. Plant Biol. 69, 789-815 (2018).

3. J. P. Lynch, Root phenotypes for improved nutrient capture: an underexploited opportunity for global agriculture. New Phytol. 223, 548-564 (2019).

4. $\quad$ K. P. Voss-Fels, R. J. Snowdon, L. T. Hickey, Designer Roots for Future Crops. Trends Plant Sci. 23, 957-960 (2018).

5. Y. Uga, et al., Control of root system architecture by DEEPER ROOTING 1 increases rice yield under drought conditions. Nat. Genet. 45, 1097-102 (2013).

6. A. P. Wasson, et al., Traits and selection strategies to improve root systems and water uptake in water-limited wheat crops. J. Exp. Bot. 63, 3485-3498 (2012).

7. R. Tsugeki, N. V. Fedoroff, Genetic ablation of root cap cells in Arabidopsis. Proc. Natl. Acad. Sci. 96, 12941-12946 (1999).

8. E. Blancaflor, J. Fasano, S. Gilroy, Mapping the Functional Roles of Cap Cells in the Response of Arabidopsis Primary Roots to Gravity. Plant Physiol. 116, 213-222 (1998).

9. H. Konnings, The Significance of the Root Cap for Geotropism. Acta Bot. Sin. 17, 203-11 (1968).

10. S. Mancuso, P. W. Barlow, D. Volkmann, F. Baluska, Actin turnover-mediated gravity response in maize root apices: gravitropism of decapped roots implicates gravisensing outside of the root cap. Plant Signal. Behav. 1, 52-8 (2006).

11. C. Wolverton, J. L. Mullen, H. Ishikawa, M. L. Evans, Root gravitropism in response to a signal originating outside of the cap. Planta 215, 153-157 (2002).

12. J. Z. Kiss, F. D. Sack, Severely Reduced Gravitropism in Dark-Grown Hypocotyls of a Starch-Deficient Mutant of Nicotiana-Sylvestris. Plant Physiol. 94, 1867-1873 (1990).

13. F. Darwin, The Statolith Theory of Geotropism. Nature 67, 571-572 (1903).

14. N. Sæther, T. H. Iversen, Gravitropism and starch statoliths in an Arabidopsis mutant. Planta 184, 491-497 (1991).

15. J. Friml, J. Wisniewska, E. Benkova, K. Mendgen, K. Palme, Lateral relocation of auxin efflux regulator PIN3 mediates tropism in Arabidopsis. Lett. to Nat. 415, 1-4 (2002).

16. G. B. Monshausen, H. E. Zieschang, A. Sievers, Differential proton secretion in the apical elongation zone caused by gravistimulation is induced by a signal from the root cap. Plant, Cell Environ. 19, 1408-1414 (1996). 
17. J. M. Fasano, et al., Changes in Root Cap pH Are Required for the Gravity Response of the Arabidopsis Root. Plant Cell 13, 907-921 (2001).

18. J. L. Mullen, H. Ishikawa, M. L. Evans, Analysis of changes in relative elemental growth rate patterns in the elongation zone of Arabidopsis roots upon gravistimulation. Planta 206, 598-603 (1998).

19. G. Huang, et al., Rice actin binding protein RMD controls crown root angle in response to external phosphate. Nat. Commun. 9, 1-9 (2018).

20. K. Boonsirichai, J. C. Sedbrook, R. Chen, S. Gilroy, P. H. Masson, ALTERED RESPONSE TO GRAVITY Is a Peripheral Membrane Protein That Modulates GravityInduced Cytoplasmic Alkalinization and Lateral Auxin Transport in Plant Statocytes. Plant Cell 15, 2612-2625 (2003).

21. J. M. Guseman, K. Webb, C. Srinivasan, C. Dardick, DRO1 influences root system architecture in Arabidopsis and Prunus species. Plant J. 89, 1093-1105 (2017).

22. I. K. Dawson, et al., Barley: A translational model for adaptation to climate change. New Phytol. 206, 913-931 (2015).

23. K. F. X. Mayer, et al., A physical, genetic and functional sequence assembly of the barley genome. Nature 491, 711-716 (2012).

24. M. Mascher, et al., A chromosome conformation capture ordered sequence of the barley genome. Nature 544, 427-433 (2017).

25. R. Bovina, S. Salvi, A collection of root architecture mutants in barley (Hordeum vulgare L.), in preparation (2021).

26. S. Wu, et al., VLN2 Regulates Plant Architecture by Affecting Microfilament Dynamics and Polar Auxin Transport in Rice. Plant Cell 27, tpc.15.00581 (2015).

27. S. Waidmann, et al., Cytokinin functions as an asymmetric and anti-gravitropic signal in lateral roots. Nat. Commun. 10, 3540 (2019).

28. C. Monat, et al., TRITEX: Chromosome-scale sequence assembly of Triticeae genomes with open-source tools. Genome Biol. 20, 1-35 (2019).

29. C. J. A. Sigrist, et al., New and continuing developments at PROSITE. Nucleic Acids Res. 41, 344-347 (2013).

30. G. Denay, G. Vachon, R. Dumas, C. Zubieta, F. Parcy, Plant SAM-Domain Proteins Start to Reveal Their Roles. Trends Plant Sci. 22, 718-725 (2017).

31. C. A. Hollender, et al., Loss of a highly conserved sterile alpha motif domain gene ( WEEP ) results in pendulous branch growth in peach trees. Proc. Natl. Acad. Sci. 115, E4690E4699 (2018).

32. K. V. Krasileva, et al., Uncovering hidden variation in polyploid wheat. Proc. Natl. Acad. Sci. U. S. A. 114, E913-E921 (2017).

33. G. K. Kirschner, Y. Stahl, M. Von Korff, R. Simon, Unique and Conserved Features of the 
Barley Root Meristem. Front. Plant Sci. 8 (2017).

34. M. I. Love, W. Huber, S. Anders, Moderated estimation of fold change and dispersion for RNA-seq data with DESeq2. Genome Biol. 15, 1-21 (2014).

35. D. J. Cosgrove, Plant expansins: Diversity and interactions with plant cell walls. Curr. Opin. Plant Biol. 25, 162-172 (2015).

36. M. Taniguchi, et al., The Arabidopsis LAZY1 Family Plays a Key Role in Gravity Signaling within Statocytes and in Branch Angle Control of Roots and Shoots. Plant Cell 29, tpc.00575.2016 (2017).

37. T. Ogura, et al., EXOCYST70A3 controls root system depth in Arabidopsis via the dynamic modulation of auxin transport (2019) https:/doi.org/http://dx.doi.org/10.1101/559187.

38. M. Hála, et al., An exocyst complex functions in plant cell growth in Arabidopsis and tobacco. Plant Cell 20, 1330-1345 (2008).

39. N. Siddiqui, J. Léon, A. A. Naz, A. Ballvora, Genetics and genomics of root system variation in adaptation to drought-stress in cereal crops. J. Exp. Bot. (2020) https:/doi.org/10.1093/jxb/eraa487.

40. W. Sinclair, I. Oliver, P. Maher, A. Trewavas, The role of calmodulin in the gravitropic response of the Arabidopsis thaliana agr-3 mutant. Planta 199, 343-351 (1996).

41. R. D. Firn, C. Wagstaff, J. Digby, The use of mutants to probe models of gravitropism. J. Exp. Bot. 51, 1323-1340 (2000).

42. A. M. Jones, et al., Border Control--A Membrane-Linked Interactome of Arabidopsis. Science (80-. ). 344, 711-716 (2014).

43. F. Passardi, C. Penel, C. Dunand, Performing the paradoxical: How plant peroxidases modify the cell wall. Trends Plant Sci. 9, 534-540 (2004).

44. J. H. Joo, Y. S. Bae, J. S. Lee, Role of Auxin-Induced Reactive Oxygen Species in. Plant Physiol. 126, 1055-1060 (2001).

45. V. Talamè, et al., TILLMore, a resource for the discovery of chemically induced mutants in barley. Plant Biotechnol. J. 6, 477-485 (2008).

46. A. Osthoff, P. Donà dalle Rose, J. A. Baldauf, H. Piepho, F. Hochholdinger, Transcriptomic reprogramming of barley seminal roots by combined water deficit and salt stress. BMC Genomics 20, 325 (2019).

47. D. C. Hoagland, D. I. Arnon, The water culture method for growing plant without soil. Coll. Agric. Univ. California. Berkeley. Circular 3 (1950).

48. K. A. Nagel, et al., GROWSCREEN-Rhizo is a novel phenotyping robot enabling simultaneous measurements of root and shoot growth for plants grown in soil-filled rhizotrons. Funct. Plant Biol. 39, 891-904 (2012).

49. D. Pflugfelder, et al., Non-invasive imaging of plant roots in different soils using magnetic 
resonance imaging (MRI). Plant Methods 13, 1-9 (2017).

50. C. A. Schneider, W. S. Rasband, K. W. Eliceiri, NIH Image to ImageJ: 25 years of image analysis. Nat. Methods 9, 671-675 (2012).

51. A. Lampropoulos, et al., GreenGate - A Novel, Versatile, and Efficient Cloning System for Plant Transgenesis. PLoS One 8, e83043 (2013).

52. A. Hecker, et al., Binary 2in1 vectors improve in planta (Co)localization and dynamic protein interaction studies. Plant Physiol. 168, 776-787 (2015).

53. R. W. Michelmore, I. Paran, R. V Kesseli, Identification of markers linked to diseaseresistance genes by bulked segregant analysis: a rapid method to detect markers in specific genomic regions by using segregating populations. Proc. Natl. Acad. Sci. 88, 9828-9832 (1991).

54. J. Comadran, et al., Natural variation in a homolog of Antirrhinum CENTRORADIALIS contributed to spring growth habit and environmental adaptation in cultivated barley. Nat. Genet. 44, 1388-1391 (2012).

55. D. L. Hyten, et al., High-throughput genotyping with the GoldenGate assay in the complex genome of soybean. Theor. Appl. Genet. 116, 945-952 (2008).

56. H. Li, R. Durbin, Fast and accurate short read alignment with Burrows-Wheeler transform. Bioinformatics 25, 1754-1760 (2009).

57. H. Li, et al., The Sequence Alignment/Map format and SAMtools. Bioinformatics 25, 20782079 (2009).

58. H. Li, A statistical framework for SNP calling, mutation discovery, association mapping and population genetical parameter estimation from sequencing data. Bioinformatics 27, 2987-2993 (2011).

59. P. Cingolani, et al., A program for annotating and predicting the effects of single nucleotide polymorphisms, SnpEff. Fly (Austin). 6, 80-92 (2012).

60. D. van Dusschoten, et al., Quantitative 3D analysis of plant roots growing in soil using magnetic resonance imaging. Plant Physiol. 170, 1176-1188 (2016).

61. N. Kumar, et al., Further analysis of barley MORC1 using a highly efficient RNA-guided Cas9 gene-editing system. Plant Biotechnol. J. 16, 1892-1903 (2018).

62. G. R. Lazo, P. A. Stein, R. A. Ludwig, A DNA Transformation-Competent Arabidopsis Genomic Library in Agrobacterium. Bio/Technology 9, 963-967 (1991).

63. J. Imani, L. Li, P. Schäfer, K.-H. Kogel, STARTS - A stable root transformation system for rapid functional analyses of proteins of the monocot model plant barley. Plant J. 67, 726735 (2011).

64. M. W. Pfaffl, A new mathematical model for relative quantification in real-time RT-PCR. Nucleic Acids Res. 29, 45e - 45 (2001).

65. M. S. Anjam, et al., An improved procedure for isolation of high-quality RNA from 
nematode-infected Arabidopsis roots through laser capture microdissection. Plant Methods 12, 1-9 (2016).

66. A. Matei, et al., How to make a tumour: cell type specific dissection of Ustilago maydisinduced tumour development in maize leaves. New Phytol. 217, 1681-1695 (2018).

67. A. M. Bolger, M. Lohse, B. Usadel, Trimmomatic: A flexible trimmer for Illumina sequence data. Bioinformatics 30, 2114-2120 (2014).

68. A. Dobin, et al., STAR: Ultrafast universal RNA-seq aligner. Bioinformatics 29, 15-21 (2013).

69. S. Anders, P. T. Pyl, W. Huber, HTSeq-A Python framework to work with high-throughput sequencing data. Bioinformatics 31, 166-169 (2015).

70. Y. Benjamini, Y. Hochberg, Controlling the False Discovery Rate $\square$ : A Practical and Powerful Approach to Multiple Testing. R. Stat. Soc. 57, 289-300 (1995).

71. T. Tian, et al., AgriGO v2.0: A GO analysis toolkit for the agricultural community, 2017 update. Nucleic Acids Res. 45, W122-W129 (2017).

72. S. Kumar, G. Stecher, M. Li, C. Knyaz, K. Tamura, MEGA X: Molecular evolutionary genetics analysis across computing platforms. Mol. Biol. Evol. 35, 1547-1549 (2018).

73. Masatoshi Nei and Sudhir Kumar, Molecular Evolution and Phylogenetics (Oxford University Press, 2000).

74. D. T. Jones, W. R. Taylor, J. M. Thornton, The rapid generation of mutation data matrices from protein sequences. Bioinformatics 8, 275-282 (1992). 


\section{Figures and Tables}

\section{Figure 1: Root phenotype of egt2-1}

A Wild type and egt2-1 roots grown on germination papers, 7 days after germination (DAG). Scale bar: $2 \mathrm{~cm}$.

B Wild type and egt2-1 roots grown in rhizotrons 26 DAG; scale bar: $10 \mathrm{~cm}$.

C MRI pictures of wild type and egt2-1 plants grown in soil 3 DAG. Scale bar: $4 \mathrm{~cm}$.

D Root angle of seminal roots 7 DAG; $n=40$ per genotype in one experiment; two-tailed t-test, ** $p<0.01$.

E Lateral root angle 14 DAG; $n=8-9$ per genotype in two independent experiments; two-tailed ttest, ${ }^{* *} p<0.01$.

F Wild type and egt2-1 roots after rotation (time point 0) at indicated time points. Scale bar:1 cm.

G Root tip angle after rotation; plants 5 DAG were rotated by $90^{\circ}$ (time 0 ) and the root tip angle was measured over time; $n=38$ per genotype in three independent experiments; the two genotypes were compared between each other at the respective time points by a two-tailed t-test, ${ }^{* *} p<0.01$; standard deviation is depicted; to account for the different starting angles of the roots, all measurements were normalized to the starting angle of the roots at time 0 . 


\title{
Figure 2: EGT2 encodes a STERILE ALPHA MOTIF protein
}

\begin{abstract}
A Association of SNP markers with seminal root angle across the barley genome as established by bulk segregant analysis (BSA) in the $\mathrm{F}_{2}$ cross TM2835 (egt2-1, hypergravitropic roots) $\times \mathrm{CV}$. Barke (wt roots). $Y$ axis reports $\Delta \theta$, an index accounting for the difference in allele-specific fluorescence signal between the two BSA DNA bulks, per SNP.
\end{abstract}

B Gene structure of EGT2 (HORVU.MOREX.r2.5HG0370880.1) with mutations in egt2 (egt2-1: G to $A$ transition and egt2-2_deletion); exons depicted as gray box, introns depicted by lines, UTRs depicted as white boxes; red box indicates the sequence encoding for the SAM domain.

C Exemplary pictures of wild type (cv. Golden Promise) and mutant egt2-2 roots 7 DAG. Scale bar: $2 \mathrm{~cm}$.

D Seminal root angle of wild type (cv. Golden Promise) and mutant egt2-2 7 DAG; $n=15-17$ in two independent experiments.

E Root angle of lateral roots 14 DAG; $n=16-18$ in two independent experiments; two-tailed t-test, ${ }^{*} p<0.05,{ }^{* *} p<0.01$.

F Exemplary pictures of wheat wild type (WTMT) and egt2 (mut/mut) roots, 7 days after germination (DAG). Scale bar: $1 \mathrm{~cm}$.

G Root angle between $2^{\text {nd }}$ and $3^{\text {rd }}$ seminal root of wild type (WT/WT) and egt2 (mut/mut) wheat seedling at 7 DAG; $n=18$ and 39 for wt and mutant, respectively. Wheat plants were derived from two independent segregating populations. 


\section{Figure 3: Expression of EGT2}

A RNA in situ hybridization of EGT2; negative controls (sense probes) are shown on the right. Scale bar: $200 \mu \mathrm{m}$.

B FPM normalized values for EGT2 expression from the RNAseq dataset in the respective tissues (compare tissue Figure 4D); ${ }^{*} p_{a d j}<0.05$.

C qRT-PCR of EGT2 expression; normalized to tubulin; two-tailed t-test does not display significant differences.

D EGT2 localization in tobacco. Scale bar: $50 \mu \mathrm{m}$. 
Figure 4. RNAseq reveals differences in cell wall-related processes in the elongation zone

A Wild type and egt2-1 plants 3 DAG used for RNA isolation. Scale bar: $1 \mathrm{~cm}$; arrow heads point to exemplary roots used for RNA isolation (most vertical ones).

B Principal component analysis (PCA) of the 24 RNA-seq samples of the two genotypes and three tissues; first and second principal components collectively explain $82 \%$ of the variance. C Venn diagram showing upregulated (upward arrow) and downregulated (downward arrow) differentially expressed genes (DEGs) in the respective tissue.

D Experimental setup: RNA of root cap, meristem and $900 \mu \mathrm{m}$ of the elongation zone were isolated.

E Enriched gene ontology (GO) terms for DEGs downregulated in the elongation zone. 


\section{Supplementary Figure 1: Root phenotype of egt2-1}

A Wild type and egt2-1 roots grown on paper sheets, 14 days after germination (DAG). Scale bar: $2 \mathrm{~cm}$.

B Number of seminal roots 7 DAG; $n=40$ per genotype in one experiment.

C Root length 14 DAG; $n=8-9$ per genotype in two independent experiments.

D Root length after rotation; $\mathrm{n}=32$ in three independent experiments; compare Figure 1G; standard deviation is depicted; two-tailed t-test did not show any significant differences between the genotypes at respective time points; all measurements were normalized to the starting length of the roots at time point 0 .

E Magnetic resonance imaging (MRI) pictures of wild type and egt2-1 roots grown in soil for 7 DAG. Scale bar: $2 \mathrm{~cm}$.

F Root angle of roots grown in soil for 3 DAG and captured by MRI (see D); $n=17-18$ per genotype; two-tailed t-test, ${ }^{* *} p<0.01$.

G Root angle of seminal roots of plants grown in rhizotrons, measured by angle between the outermost seminal roots; 26 DAG; $\mathrm{n}=10-11$ per genotype; two-tailed t-test, ${ }^{* *} p<0.01$.

$\mathbf{H}$ Root angle of lateral roots of plants grown in rhizotrons, angle measured between main roots and lateral roots; 26 DAG: $\mathrm{n}=5$ (30 lateral roots per plant) per genotype; two-tailed t-test, ${ }^{* *} p$ $<0.01$. 


\section{Supplementary Figure 2: Meristem phenotype of egt2-1 resembles the wild type phenotype}

A Root meristem of wild type and egt2-1 7 DAG; arrow heads mark transition to elongation zone. Scale bar: $200 \mu \mathrm{m}$.

B Wild type and egt2-1 root cap 7 DAG. Scale bar: $50 \mu \mathrm{m}$; brightness adjusted in magnification.

C Quantification of meristem length 7 DAG; $n=15-20$ roots per genotype; two-tailed t-test does not show any significant difference.

D Lateral roots of wild type and egt2-1 plants 14 DAG stained by mPS-PI staining. Scale bar: $50 \mu \mathrm{m}$. 


\section{Supplementary Figure 3: The egt2-1 mutant and the corresponding wild type react similarly to auxin treatment}

A, C, E, G, I Angle of the root tip after indicated treatment and rotation for $90^{\circ}$ (time point 0 ).

$\mathbf{B}, \mathbf{D}, \mathbf{F}, \mathbf{H}, \mathbf{J}$ Length of the roots after indicated treatment and rotation for $90^{\circ}$ (time point 0 ); the two genotypes were compared between each other at the respective time points by a two-tailed ttest, ${ }^{*} p<0.05$; standard deviation is depicted; to account for the different starting angles of the roots, all measurements were normalized to the starting angle/length of the roots at time point 0 .

A, B IAA treatment; $\mathrm{n}=4-6$ per genotype and treatment in two independent experiments.

C, D NAA treatment; $n=4-6$ per genotype and treatment in two independent experiments.

E, F 2,4D treatment; $n=5-10$ per genotype and treatment in three independent experiments.

G, H NPA treatment; $\mathrm{n}=7-10$ per genotype and treatment in three independent experiments.

I, J Cytokinin treatment; $n=4-8$ per genotype and treatment in two independent experiments 


\section{Supplementary Figure 4: CRISPR/Cas9 induced mutation in EGT2 and conserved function in wheat}

A Gene model of EGT2 and partial DNA sequence; arrow marks translation start site; purple boxes mark the CRISPR target sites; in the egt2-2 mutant line, CRISPR/Cas induced a deletion between the target sites as depicted.

B Protein structure of EGT2 with the SAM domain from amino acid 172 - 225; protein alignment of EGT2, AtSAM5 (At3g07760) and peach WEEP (Prupe. 3G200700) in the SAM region.

C Root length 7 DAG, two-tailed t-test does not show a significant difference $(p<0.05) ; \mathrm{n}=15-17$ in two independent experiments.

D Wild type GP and egt2-2lateral roots 14 DAG. Scale bar: $1 \mathrm{~cm}$.

E Root tip angle after rotation; plants 5 DAG were rotated by $90^{\circ}$ (time 0 ) and the root tip angle was measured over time; $n=20$ per genotype in two independent experiments; the two genotypes were compared between each other at the respective time points by a two-tailed t-test and no significant difference was detected; standard deviation is depicted; to account for the different starting angles of the roots, all measurements were normalized to the starting angle of the roots at time 0 .

F Root length after rotation as described in $\mathrm{D} ; \mathrm{n}=20$ per genotype in two independent experiments.

G Area enclosed by the first three seminal root of wild type (WT/WT) and egt2 (mut/mut) wheat seedling at 7 DAG; $n=16$ and 35 for wild type and mutant, respectively.

H Number of seminal roots of wild type (WTMT) and egt2 (mut/mut) wheat seedling at 7 DAG; $\mathrm{n}$ $=18$ and 39 for wild type and mutant, respectively.

I Length of the first three seminal root in wild type (WT/WT) and egt2 (mut/mut) wheat seedling at 7DAG; $\mathrm{n}=16$ and 35 for wt and mutant, respectively.

Wheat plants analyzed in $\mathrm{G}, \mathrm{H}$ and I were derived from two independent segregating populations; the two genotypes were compared by a two-tailed t-test ( ${ }^{*} p<0.05$, ns $=$ no significant difference). 
bioRxiv preprint doi: https://doi.org/10.1101/2021.01.23.427880; this version posted January 23, 2021. The copyright holder for this preprint

(which was not certified by peer review) is the author/funder, who has granted bioRxiv a license to display the preprint in perpetuity. It is made available under aCC-BY-NC 4.0 International license.

\section{Supplementary Figure 5: Phylogenetic tree of EGT2 and related proteins}

Species abbreviations: AT: Arabidopsis thaliana; BGIOS: Oryza sativa Indica Group; BRADI: Brachypodium dystachyon; Os: Oryza sativa Japonica Group; PRUPE: Prunus persica; SORBI: Sorghum bicolor; Traes: Triticum aestivum; TRITD: Triticum durum; Zm: Zea mays. 
bioRxiv preprint doi: https://doi.org/10.1101/2021.01.23.427880; this version posted January 23,2021 . The copyright holder for this preprint (which was not certified by peer review) is the author/funder, who has granted bioRxiv a license to display the preprint in perpetuity. It is made available under aCC-BY-NC 4.0 International license.

Supplementary Figure 6: Heat map of differentially expressed genes (FDR $<5 \%$ and log2FC $>|2|$ ) between wild-type and egt2-1 in root cap, root meristem and elongation zone

Average of $\log 1 p$ of fpm normalized counts of four biological replicates of the respective genotype and tissue is shown in blue scale. 
bioRxiv preprint doi: https://doi.org/10.1101/2021.01.23.427880; this version posted January 23, 2021. The copyright holder for this preprint (which was not certified by peer review) is the author/funder, who has granted bioRxiv a license to display the preprint in perpetuity. It is made available under aCC-BY-NC 4.0 International license.

\section{Supplementary Figure 7: Expression of expansins}

Fpm normalized expression values of the expansins differentially expressed in the gene set; padj is depicted with * $p<0.05,{ }^{* *} p<0.01 ; \mathrm{RC}$ : root cap, M: meristem, EZ: elongation zone. 


\section{Supplementary Table 1. BSA mapping results}

\section{Supplementary Table 2. SNPs whole genome sequencing}

Supplementary Table 3. Overview of RNAseq reads and mapping results

\section{Supplementary Table 4: DEGs}

Supplementary Table 5: primer sequences 


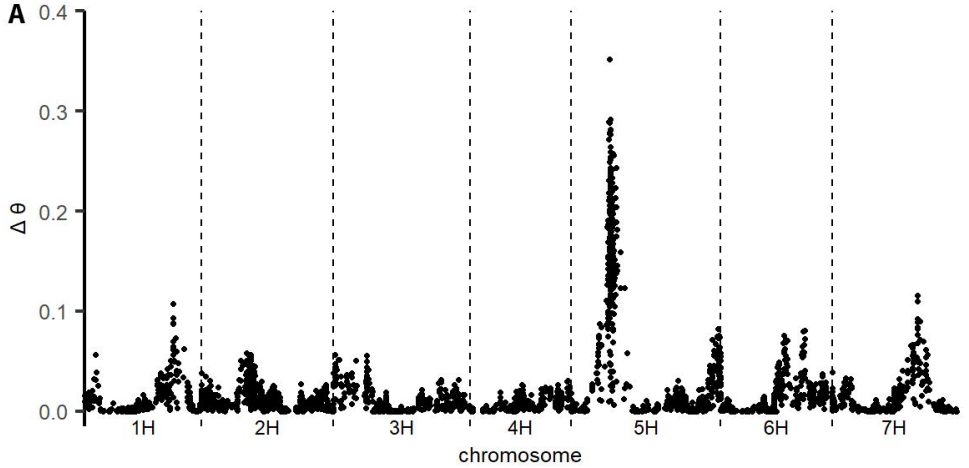

B
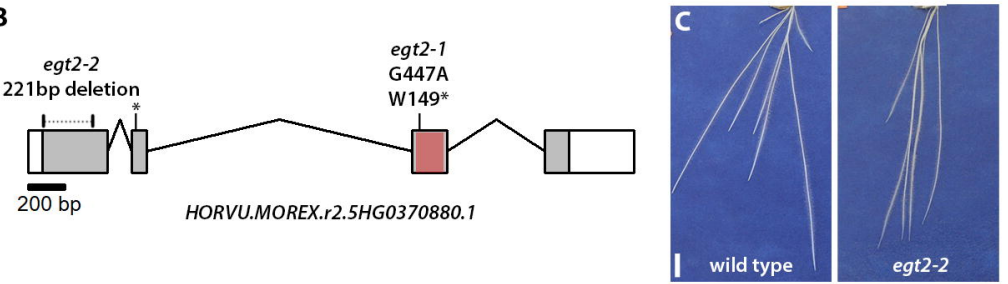

足
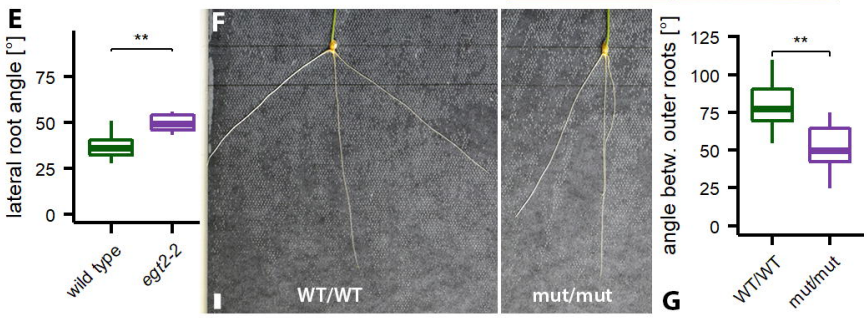


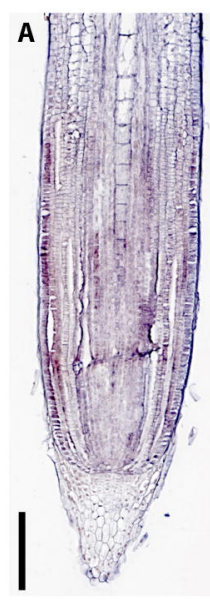

anti sense

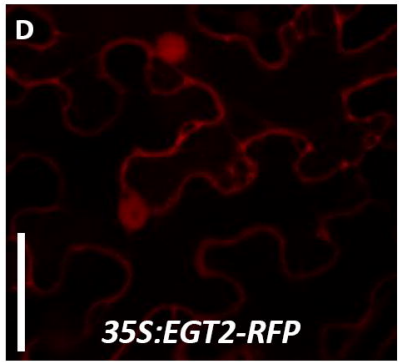

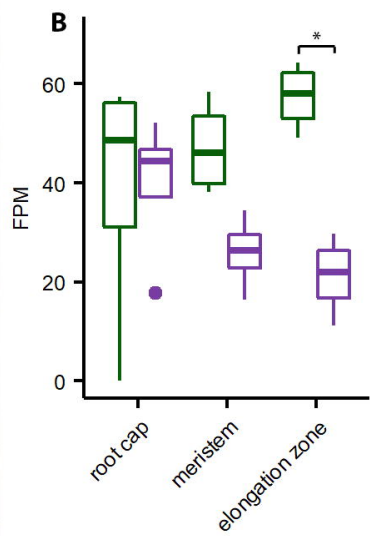

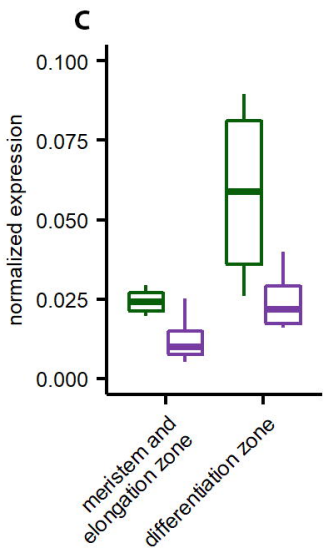

wild type

$\underbrace{\prime}$ egt2-1

\section{sense}

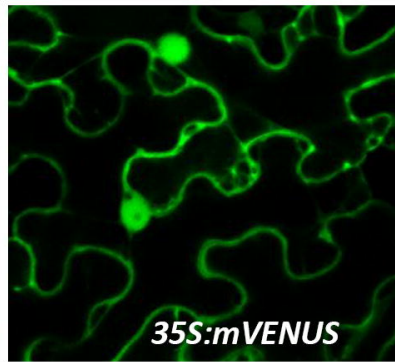

DAPI

bright fiold 
